\title{
An interferometric radar for remote sensing of deflections on large structures
}

\author{
C. Gentile, S. Bulgarelli, N. Gallino \& A. Oldini \\ Politecnico di Milano, Dept. of Structural Engineering, Italy
}

\begin{abstract}
Recent progresses in radar techniques have favoured the development of microwave interferometers, suitable for the non-contact monitoring of large structures. The main characteristic of the new radar systems - entirely designed and developed by Italian researchers - is the possibility of simultaneously measuring the static or dynamic deflection of several points on a large structure with high accuracy.

In this paper, some techniques implemented in the microwave interferometers are summarized in order to highlight advantages and potential issues of the radar-based measurement; afterwards, the accuracy and simplicity of the radar technology is demonstrated in applications to full-scale bridges.
\end{abstract}

Keywords: bridge, cable, microwave interferometry, static tests, radar.

\section{Introduction}

The development of innovative non-contact systems for vibration measurement has recently drawn the attention of several researchers. Examples of non-contact sensors include Laser Doppler Vibrometer (Cunha and Coetano [1], Casadei et al. [2]), Global Positioning Systems (Nickitopoulou et al. [3]) and vision-based systems using digital image processing techniques (Lee and Shihozuka [4]).

In addition, the microwave interferometry has recently emerged as an innovative technology, suitable to the non-contact vibration monitoring of large structures. New radar systems - entirely designed and developed by Italian researchers (IDS, Ingegneria Dei Sistemi, Pisa, Italy) - are now available and capable of simultaneously measuring the static or dynamic deflection of several points on a large structure with high accuracy (Bernardini et al. [5], Gentile and Bernardini [6]). 
After the construction of some prototypes of the new non-contact vibrometer, named IBIS-S (Image By Interferometric Survey of Structures), a joint research started between IDS and the Politecnico di Milano (Dept. of Structural Engineering), mainly aimed to validate the results of the equipment and to assess its performance in ambient vibration testing (AVT) of bridges. The first results of this investigation are reported in (Bernardini et al. [5], Gentile and Bernardini [6]) and included:

1. establishing the actual displacement sensitivity of the equipment $(<0.02 \mathrm{~mm})$ through free vibration tests carried out in the laboratory on a simple springmass system;

2. verifying the stability in long term functioning (both in the laboratory and onsite), required for effective employment in AVT or continuous dynamic monitoring;

3. AVT of a reinforced concrete bridge, using simple radar reflectors placed as close as possible to conventional sensors. The velocity time-histories evaluated from the radar sensor (and obtained by deriving the displacements) generally exhibited excellent agreement with the ones recorded by conventional sensors. Furthermore, resonant frequencies and mode shapes of the bridge, that were identified from the radar signals, turned out to be as accurate as that obtained with traditional accelerometers.

In the paper, some concepts of the main radar techniques adopted in the radar-based measurement of deflections are first summarized, in order to highlight advantages and potential issues of the new technology and to properly discuss the technical characteristics of the IBIS-S sensor. Subsequently, the accuracy and simplicity provided by the radar techniques in the application to static test of a steel-composite bridge and to vibration survey of the cables of a cable-stayed bridge are presented and discussed.

\section{The radar measurement system}

The radar sensor used in this work (IDS, IBIS-S system) is an industrially engineered micro-wave interferometer and consists of a sensor module, a control $\mathrm{PC}$ and a power supply unit.

The sensor module (Fig. 1) is a coherent radar (i.e. a radar preserving the phase information of the received signal) generating, transmitting and receiving the electromagnetic signals to be processed in order to provide the deflection measurements. The equipment radiates at a central frequency of $16.75 \mathrm{GHz}$ so that the radar is classified as $\mathrm{Ku}$-band, according to the standard radar-frequency letter-band nomenclature from IEEE Standard 521-1984. The sensor unit, weighing $12 \mathrm{~kg}$, is installed on a tripod equipped with a rotating head, allowing the sensor to be orientated in the desired direction, as shown in Fig. 1. The module has an USB interface for connection with the control PC and an interface for the power supply module; in addition, two horn antennas are placed on the sensor module for transmission and reception of the electromagnetic waves. 


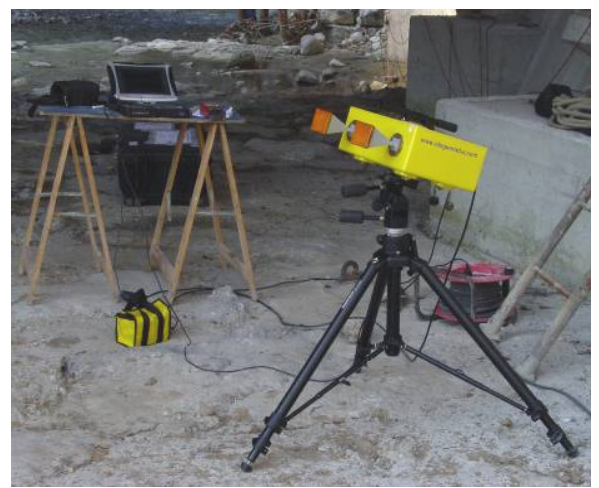

Figure 1: $\quad$ View of the IBIS-S microwave interferometer.

The control PC is provided with the software for the system management and is used to configure the acquisition parameters, store the acquired signals, process the data and view selected results in real time.

Finally, the power supply unit provides power to the system through a $12 \mathrm{~V}$ battery unit.

The main functions of the equipment are the simultaneous detection of the position and deflection of different targets placed at different distances from the sensor. This performance is obtained by using two well-known radar techniques:

1. the Stepped-Frequency Continuous Wave (SF-CW) technique (Wehner [7]), implemented to resolve the scenario in the range direction, i.e. to detect the position of different target surfaces placed along the radar's line of sight;

2. the phase Interferometry technique (Henderson and Lewis [8]) adopted to compute the displacement of each target by comparing the phase information of the back-scattered electromagnetic waves collected at different times.

A SF-CW radar continuously transmits bursts of $N$ electromagnetic pulses, generally named tones, whose frequencies are increased from tone to tone by a constant frequency increment $\Delta f$; hence, a large effective bandwidth $B=(N-1) \Delta f$ is attained. A large value of $B$ is, in turn, highly desirable since the range resolution (i.e. the minimum separation that can be detected along the radar's line of sight) $\Delta r$ may be expressed as:

$$
\Delta r=\frac{c}{2 B}
$$

where $c$ is the speed of light in free space.

Furthermore, the use of step-frequency continuous waveform exhibits various advantages: (a) $\mathrm{CW}$ radars can reach the same far distance of a pulse radar by transmitting lower power and, in turn, low transmitted power generally allows SF-CW radars to be included in the Short Range Device category as a licensefree use equipment; (b) SF modulated radars can transmit and receive signals with precise frequency control by using Direct Digital Synthesizer, an innovative up-to-date device for generating step-frequency signals. 
In a SF-CW radar, the signal source dwells at each frequency $f_{k}=f_{o}+k \Delta f$ $(k=0,1,2, \ldots, N-1)$ long enough to allow the received echoes to reach the receiver. Hence, the duration of each single pulse $\left(T_{\text {tone }}\right)$ depends on the maximum distance $\left(R_{\max }\right)$ to be observed in the scenario:

$$
T_{\text {tone }}=\frac{2 R_{\max }}{c}
$$

The number $N$ of tones composing each burst can be computed as:

$$
N=\frac{2 R_{\max }}{\Delta r}
$$

The time scheduling (2) permits the SF-CW radar to receive the response of the furthest target before transmitting the following tone. On the other hand, Eqs. (2)-(3) clearly highlight that the maximum sampling rate of the scenario $f_{\text {sample }}$ depends on $R_{\max }$ and $\Delta r$. Specifically, accounting for Eqs. (2)-(3), $f_{\text {sample }}$ can be expressed as:

$$
f_{\text {sample }} \cong \frac{1}{N T_{\text {tone }}}=\frac{c}{2 N R_{\max }}=\frac{c \Delta r}{4 R_{\max }^{2}}
$$

Eq. (4) clearly shows that: (a) the maximum sampling rate decreases as the maximum measured distance increases; (b) the maximum sampling rate increases as the range resolution increases.

The magnitude of the IDFT of the received echoes at each time sample provides a synthetic profile (i.e. a 1-D map) of the scattering objects in the space illuminated by the antenna beam, as a function of their relative distance from the sensor. Fig. 2 shows an ideal range profile, as expected when the radar beam illuminates a series of targets at different distances and different angles from the axis of the system. The peaks in plot of Fig. 2 correspond to points with good

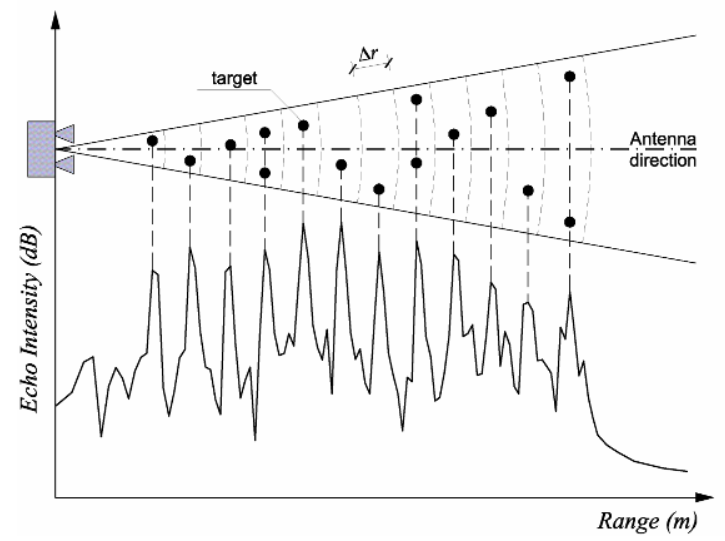

Figure 2: $\quad$ View of the IBIS-S microwave interferometer. 


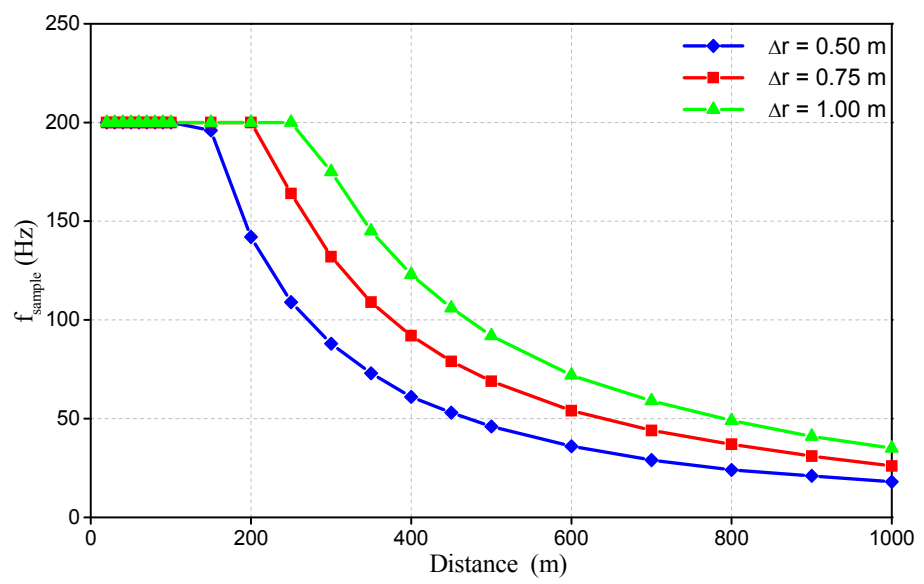

Figure 3: Sampling rate vs. maximum distance for three different values of the range resolution.

electromagnetic reflectivity; the position of these targets is identified from the range (i.e. distance from the sensor) and the radar can simultaneously detect the displacement or the transient response of these points. Fig. 2 also shows that the radar has only 1-D imaging capabilities, i.e. different targets can be individually detected if they are placed at different distances from the radar (Gentile et al. [9]).

The IBIS-S sensor was designed to provide a minimum range resolution of $0.50 \mathrm{~m}$, so that two targets can still be detected individually if their relative distance is greater than or equal to $0.50 \mathrm{~m}$.

The dependence of sampling rate on the maximum distance is shown in Fig. 3 for three different distance resolutions. Fig. 3 clearly highlights that, for a range resolution of $0.5 \mathrm{~m}$, the sampling rate drops off for distances larger than $150.0 \mathrm{~m}$ while, for a range resolution of $1.0 \mathrm{~m}$, the sampling rate starts to decrease for distances exceeding $300.0 \mathrm{~m}$ and reaches the value of $35 \mathrm{~Hz}$ for a range of $1000.0 \mathrm{~m}$. Fig. 3 also shows that the maximum value of the sampling rate has been limited to $200 \mathrm{~Hz}$ because the significant frequency content of the displacement time-histories is generally in the frequency range $0-20 \mathrm{~Hz}$ for a large structure; in addition, sampling interval $\Delta t=0.005 \mathrm{~s}$ is in principle well suitable to provide a good waveform definition of the acquired signals.

According to phase interferometry technique in radar systems, when the electro-magnetic wave is reflected from a target moving along the direction of wave propagation, a phase shift arises between the signals reflected by the target surface at different times. In other words, the phase of a signal received from a target depends, on its distance so that the occurred variation of distance can be determined by evaluating the phase differences of range profiles obtained at different times. 
Since the interferometric technique provides a measurement of the radial displacement, the evaluation of the actual displacement requires the prior knowledge of the direction of motion.

The main technical and operational characteristics of IBIS-S are summarized as follows:

1. minimum range resolution: $0.50 \mathrm{~m}$;

2. maximum sampling frequency: $200 \mathrm{~Hz}$;

3. maximum operating distance: depending on $f_{\text {sample }}$ and $\Delta r$ (Fig. 3).

\section{Static tests of a steel-composite arch bridge}

\subsection{Description of the bridge}

The investigated bridge (Fig. 4), located about $75 \mathrm{~km}$ far from Milan, crosses the Pioverna creek between the municipalities of Pasturo and Introbio. Elevation and plan of the bridge, opened to traffic at the beginning of August 2008, are shown in Fig. 4.

The bridge, $38 \mathrm{~m}$ long, has skew deck and consists of 3 vertical steel arched frames transversally connected to 7 steel I-shape floor beams; the girders and floor beams are all composite with a $25.0 \mathrm{~cm}$ concrete slab. The arch configuration of each vertical frame approximates an inclined leg frame bridge with curved legs.

The deck is characterized by a longitudinal slope of $1.5 \%$ and the skew angle is approximately $60^{\circ}$.

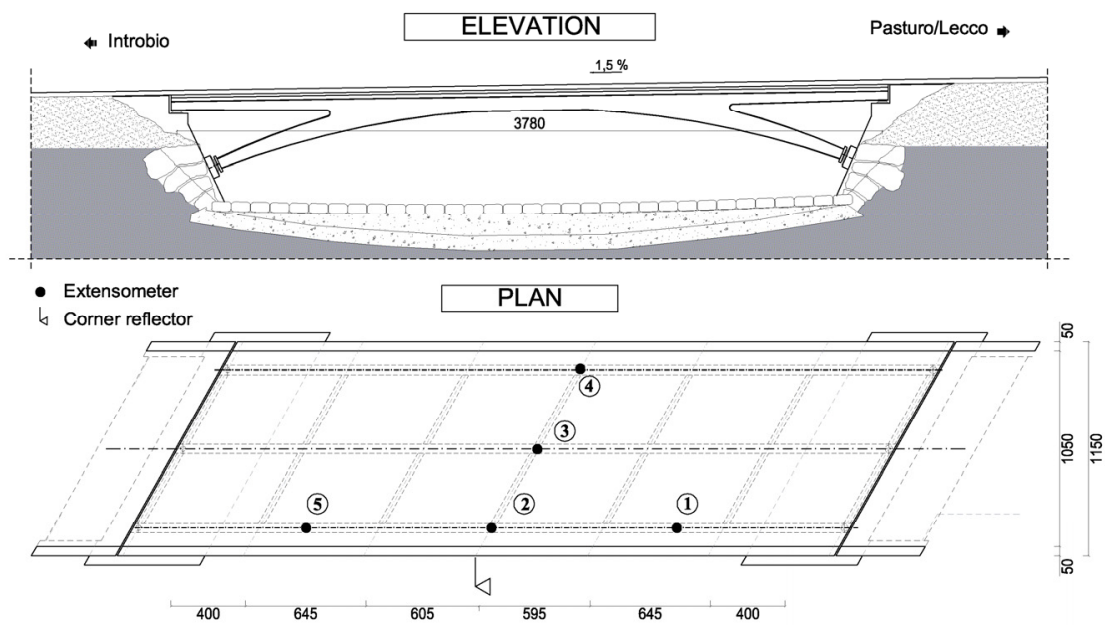

Figure 4: Elevation and plan of the bridge crossing the Pioverna Creek (dimensions in $\mathrm{cm}$ ). 


\subsection{Experimental procedures and radar results}

As it is usual in reception tests of new bridges, the focus of the test program was the measurement of vertical deflections of the bridge under live load. Vehicles of known weight were placed according to four different arrangements to provide four live load cases; vertical deflections were measured at 5 selected points (Figs. 4-5) by using traditional extensometers (Fig. 5). In addition, one passive radar reflector or corner reflector was placed on the deck, as shown in Figs. 4-6.
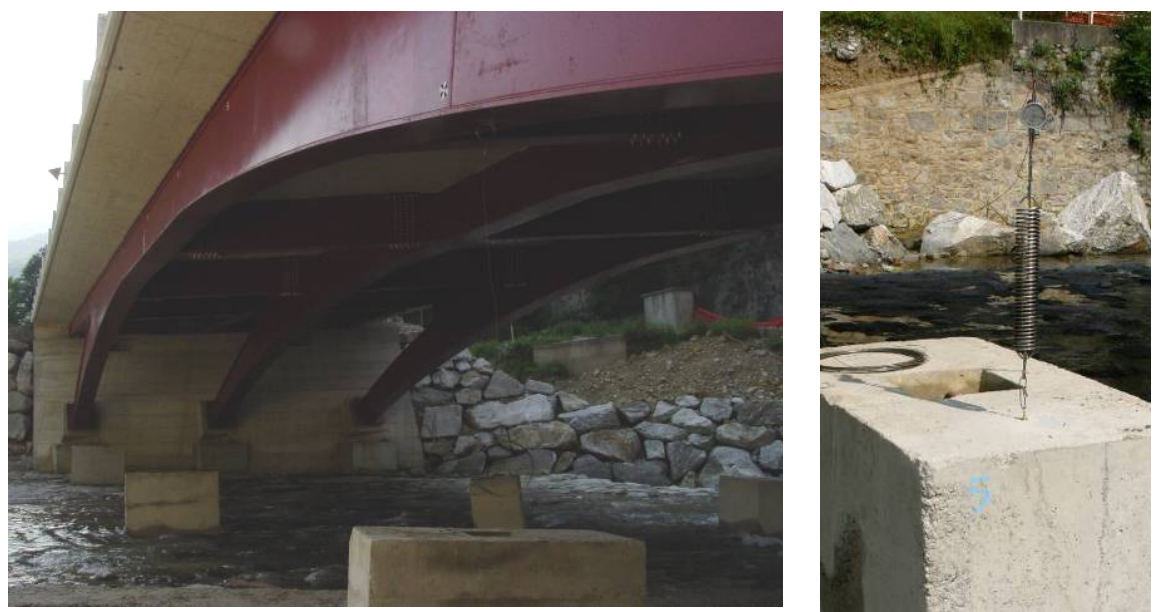

Figure 5: View of the extensometers used during the load tests.

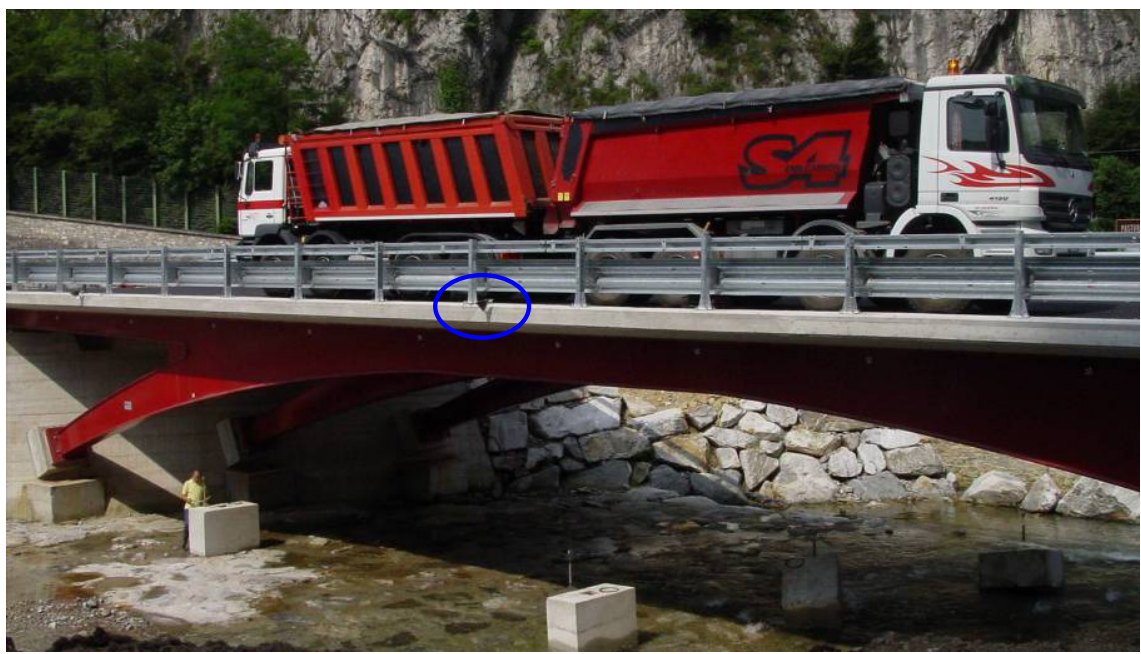

Figure 6: View of the investigated load condition and of the radar reflector. 
(a)

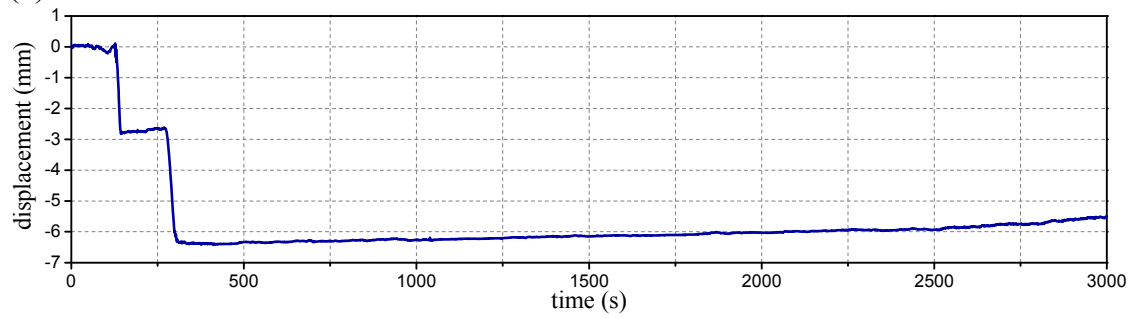

(b)

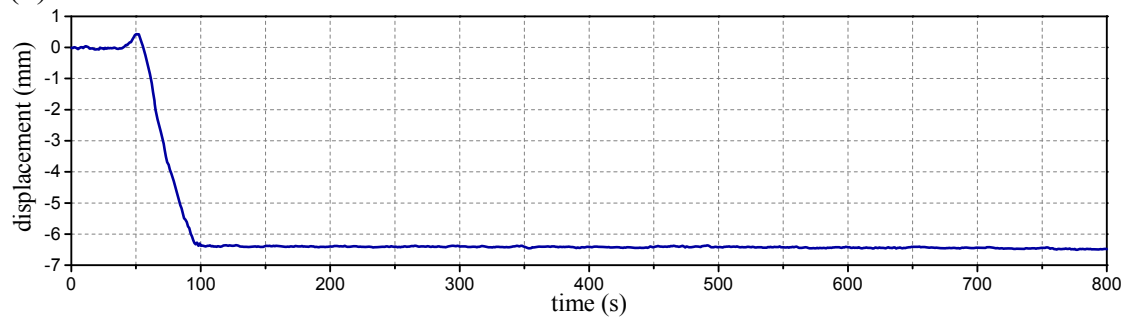

Figure 7: Progress in time of the vertical displacement measured by the radar technique in: (a) morning test and (b) late afternoon test.

Due to space limitations, only the results of the load configuration shown in Fig. 6 will be presented and discussed. This configuration, with two test vehicles eccentrically loading the deck, was repeated twice: first, during the morning, as part of a more complex load arrangement and in the late afternoon.

Figs. $7 \mathrm{a}$ and $7 \mathrm{~b}$ show the displacement time-histories obtained by the radar sensor in correspondence of the corner reflector, in the morning and in the late afternoon, respectively. The inspection of Figs. 7a-b clearly highlights that:

1. the time windows corresponding to successive entrance and motion of the test vehicles along the bridge are clearly identified. Peculiarly, in the morning test, the two test vehicles were placed on the bridge one at time in the time interval 100-320 s (Fig. 7a) while in the afternoon test the two trucks simultaneously entered in the time interval 0-100 s (Fig. 7b);

2. during the morning test, just after the vehicles stopped on the bridge, the deck began to be directly irradiated by the sun. As a consequence of the temperature effect, the vertical deflection almost linearly decreases in time (Fig. 7a) and this effect was clearly detected by all extensometers, as well;

3. the progress in time of the displacement in the late afternoon (Fig. 7b) test (with the deck being completely in shadow) is practically constant after the positioning of the test vehicles. The maximum deflection measured by the microwave interferometer in this second test was about $6.40 \mathrm{~mm}$. This value is practically equal to the one measured in morning (Fig. 7a) and to the prediction obtained by using a FE model of the bridge, that was previously adjusted based on the operational modal analysis of the bridge; 
4. it should be observed that the maximum deflection provided by the radar significantly exceeds the one measured by the closer extensometer $(4.60 \mathrm{~mm}$, extensometer 2 in Fig. 4). On the other hand, Fig. 4 shows that the corner reflector was placed more than $1.50 \mathrm{~m}$ far from extensometer 2 . In addition, the difference between the displacement of the two points was, again, very accurately predicted by the FE model of the bridge.

\section{Dynamic measurements on stay-cables}

Structural health monitoring (SHM) of cable-stayed bridges needs periodic inspection and non-destructive assessment of the stay cables since deterioration problems of these main load-carrying elements could adversely affect the longterm behaviour of the bridges. Since the main tension elements in stay cables are hidden from the view by permanent protective barriers, dynamic measurements of cable vibration are often carried out to indirectly investigate the overall health of the cables and cable-stayed bridges.

Dynamic measurements on stay cables are generally aimed at: (a) identifying the local natural frequencies and damping ratios; (b) evaluating the cable forces and monitoring the changes in these forces over time.

Usually, the tension force is obtained from cable's natural frequencies; subsequently, the knowledge of cable forces is used to check the correct distribution of the internal forces in the bridge at the end of construction, while monitoring the possible changes in stay cable forces over time may provide an efficient method for SHM.

Within this context, the application of the radar technique to the measurement of cable vibrations is especially promising in order to perform systematic dynamic assessment of stay cables in a simple and quick way. Peculiarly, compared to other techniques of remote sensing, the microwave interferometry exhibits various advantages: (1) low power transmitted; (2) higher accuracy; (3) possibility of simultaneously measuring the dynamic response of several staycables.

In addition, the possible issues (discussed in sect. 2) that may affect the radarbased measurement practically does not exist; specifically:

a. the typical position of the sensor in the survey of an array of cables is inclined upward, as schematically shown in Fig. 8; hence, only the stays are encountered along the path of the electromagnetic waves so that 1-D imaging capability is perfectly adequate to the test scenario;

b. as usual, it can assumed that the in-plane motion of the cable is orthogonal to its axis so that the actual deflection $d$ can be expressed as:

$$
d=\frac{d_{\mathrm{r}}}{\cos \left[\boldsymbol{\pi} / 2-\left(\boldsymbol{\alpha}_{c}+\boldsymbol{\alpha}_{s}\right)\right]}
$$

where $\alpha_{\mathrm{c}}$ and $\alpha_{\mathrm{s}}$ are the slope of the cable and of the sensor, respectively (Fig. 8). 


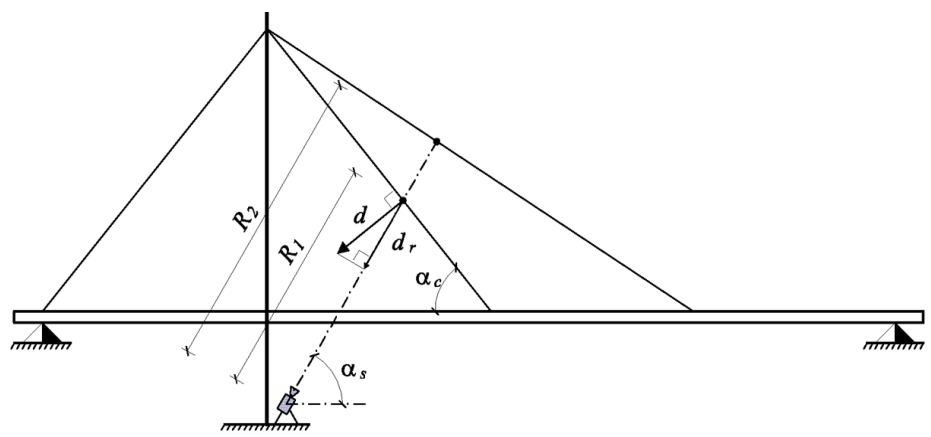

Figure 8: Radial displacement versus actual (in-plane) displacement of a cable.

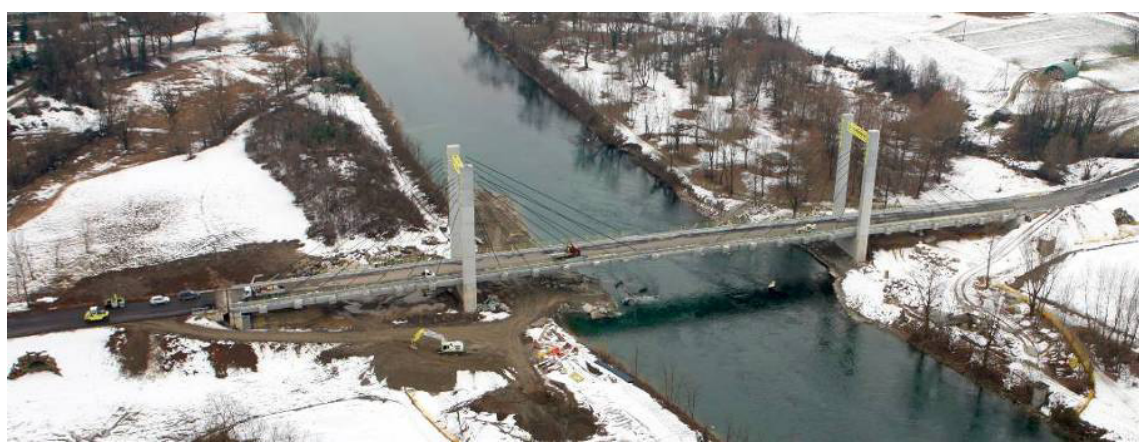

Figure 9: View of the "Cesare Cantù" cable-stayed bridge over Adda River.

Furthermore, since it is quite easy to predict the scenario under the radar beam, the inspection of the range profile allows one to quickly verify that the sensor positioning provides a correct image of the scenario.

The accuracy and operational simplicity of the radar techniques in vibration survey of stay-cables arrays has been verified on two cable-stayed bridges: some results obtained on the longer cables of the "Cesare Cantù" cable-stayed bridge are herein after presented and discussed.

\subsection{Description of the bridge}

The "Cesare Cantù" cable-stayed bridge (Fig. 9) is a roadway bridge that crosses the Adda river between the municipalities of Olginate and Calolziocorte (about $60 \mathrm{~km}$ north-east of Milan). The bridge, with a pre-stressed concrete deck, is formed by a central span of $110.0 \mathrm{~m}$ and two lateral spans of $55.0 \mathrm{~m}$.

The cast-in-place pre-stressed concrete deck is $11.50 \mathrm{~m}$ wide and consists of two two-cell box girders connected by a central slab and by a series of 24 transverse cross-beams, providing the lower anchorage of the stay cables. The deck is suspended from 24 pairs of cable stays, arranged in a semi-fan and 
connected to two $\mathrm{H}$-shaped reinforced concrete towers reaching the height of about $38.0 \mathrm{~m}$ above the foundations.

\subsection{Experimental procedures and radar results}

After the main phase of cable tensioning, vibration measurements were carried out on all cables, by using conventional accelerometers, to check the tension forces; also the global dynamic characteristics of the bridge were determined by ambient vibration tests in order to optimize the subsequent phase of adjustment of cable forces. Taking profit of these tests, dynamic measurements on some cables were carried out by simultaneously using piezoelectric accelerometers and the radar system, in order to verify the reliability and accuracy of the radar technique.

Figs. 10a-b show the auto-spectral densities (ASD) associated to the ambient response of cable S02, obtained from the measurements (at different points) of accelerometer and radar vibrometer. Although the ASDs of Figs. 10a-b are associated to different mechanical quantities measured (acceleration and displacement) and to different points of the same cable, the spectral plots clearly highlight an excellent agreement in terms of local natural frequencies of the cable and are characterized by 12 equally spaced and well-defined peaks in the frequency range $0-35 \mathrm{~Hz}$; in addition, the peaks related to the cable natural frequencies are much more evident in the ASD of radar data in the highfrequency range $(7-35 \mathrm{~Hz})$.

(a)

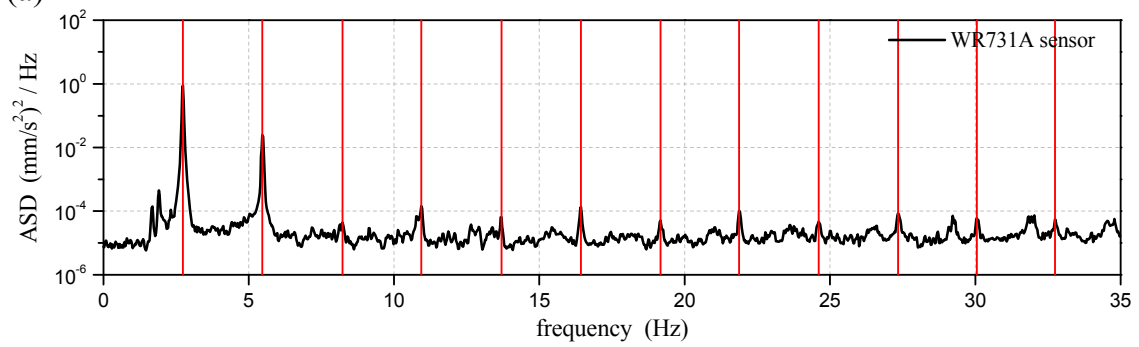

(b)

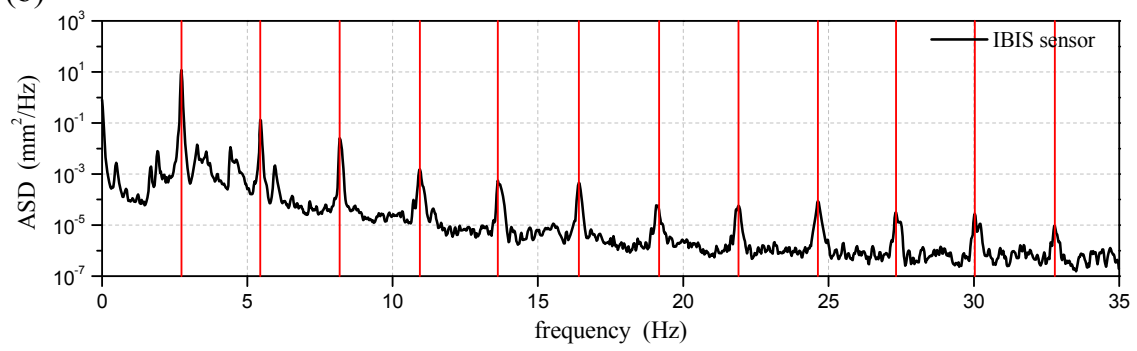

Figure 10: (a) ASD of the acceleration data recorded on cable S02; (b) ASD of the displacement data measured by the radar on cable $\mathrm{S}_{02}$. 


\section{Conclusions}

An innovative radar sensor, developed for remote (non contact) measurements of deflections on bridges and large structures in both static and dynamic conditions, has been presented in the paper. The new sensor exhibits various operational advantages with respect to contact sensors and, at the same time, provides a direct measurement of displacement, which is of utmost importance in the inservice monitoring of large structures.

The accuracy of the sensor has been evaluated in laboratory tests carried out on a simple mass-spring system; the free-vibration tests carried out on the oscillator confirm that the displacement sensitivity is better than $2 / 100 \mathrm{~mm}$; in addition, the laboratory tests indicated both an excellent quality for the measured displacements and a good operating stability of the equipment.

The use of the radar interferometer in static and dynamic tests of full-scale bridges exhibited excellent stability, when employed on site for long time intervals.

Furthermore, the radar technique turned out to be especially suitable to vibration survey of stay cables since it allows one to simultaneously measure the dynamic response of several stay-cables and provides measurements of high level of accuracy in terms of identification of natural frequencies.

\section{Acknowledgements}

The research was supported by the M.I.U.R. (Italian Ministry of University and Research), within a National Project funded in 2006, and by IDS (Ingegneria Dei Sistemi, Pisa, Italy), owner and developer of the microwave interferometer used in the tests.

The authors are indebted with Dr. Giulia Bernardini (IDS) and the Province of Lecco, owner of the tested bridges: without their involvement and cooperation, this research would not have been possible.

Special thanks are due to Marco Antico and Marco Cucchi (Laboratory of Vibrations and Dynamic Monitoring of Structures, Dept. of Structural Engineering, Politecnico di Milano), who helped the authors in conducting the field tests.

\section{References}

[1] Cunha, A. and Caetano, E. Dynamic measurements on stay cables of cablestayed bridges using an interferometry laser system. Experimental Techniques, 23(3), pp. 38-43, 1999.

[2] Casadei, P. et al. NTD monitoring of bridges using innovative high precision surveying system, Proc. IABSEE Symposium, 2006.

[3] Nickitopoulou, A., Protopsalti, K. and Stiros, S. Monitoring dynamic and quasi-static deformations of large flexible engineering structures with GPS: Accuracy, limitations and promises. Engineering Structures, 28(10), pp. 1471-1482, 2006. 
[4] Lee, J.J. and Shinozuka, M. A vision-based system for remote sensing of bridge displacement. NDT\&E International, 39(5), pp. 425-431, 2006.

[5] Bernardini, G. et al. Microwave interferometer for ambient vibration measurements on civil engineering structures: 1. Principles of the radar technique and laboratory tests. Proc. EVACES'07, pp. 143-152, 2007.

[6] Gentile, C. and Bernardini, G. Output-only modal identification of a reinforced concrete bridge from radar-based measurements. NDT\&E International, 41(7), pp. 544-553, 2008.

[7] Wehner, D.R. High-resolution radar. Artech House, $2^{\text {nd }}$ Ed., 1995.

[8] Henderson, F.M. and Lewis, A.J. (Eds.) Manual of Remote Sensing. Principles and Applications of Imaging Radar. Wiley \& Sons, $3^{\text {rd }}$ Ed, 1998.

[9] Gentile, C., Bernardini, G. and Ricci, P.P. Operational modal analysis of a cable-stayed bridge from conventional and radar-based measurements. Proc. Eurodyn 2008, 2008. 